\title{
De la nécessité de l'échange intellectuel
}

\section{Cécile Dauphin}

\section{(2) OpenEdition}

Journals

Édition électronique

URL : https://journals.openedition.org/clio/170

DOI : 10.4000/clio. 170

ISSN : 1777-5299

Éditeur

Belin

Édition imprimée

Date de publication : 1 novembre 2002

Pagination : 99-106

ISBN : 2-85816-641-2

ISSN : $1252-7017$

Référence électronique

Cécile Dauphin, «De la nécessité de l'échange intellectuel », Clio. Histoire, femmes et sociétés [En ligne], 16 | 2002, mis en ligne le 11 mars 2003, consulté le 24 avril 2022. URL : http://

journals.openedition.org/clio/170 ; DOI : https://doi.org/10.4000/clio.170 


\section{De la nécessité de l'échange intellectuel}

\section{Cécile DAUPHIN}

Les historiens se donnent trop rarement le loisir et les moyens de réfléchir à leurs pratiques et de passer en revue leurs propres outils. Il faut donc saluer l'initiative prise par CLIO HSF et remercier les organisatrices de cette journée. Dans l'esprit de conquête qui habite le champ de l'histoire des femmes depuis trois décennies, le besoin de jalonner le parcours devenait presque une habitude. De Saint-Maximin à Rouen en passant par Jussieu', des lieux ont marqué et scandé la réflexion sur l'émergence des femmes dans l'historiographie et sur la pertinence du concept de genre. L'originalité de la rencontre de Lyon est de cibler un mode spécifique de diffusion de la recherche, la revue, et de s'interroger sur son impact dans le processus de légitimation et de reconnaissance de ce champ.

"L'histoire des femmes en revues ", l'intitulé de la journée joue sur la polysémie du terme " revue " : il ne désigne pas seulement la publication périodique et scientifique, le mot laisse aussi entendre l'opération technique d'examen, d'inventaire pour donner un aperçu de différentes opinions. La connotation événementielle de parade (passer en revue) suppose également l'inspection de la bonne marche d'un ensemble : faire la part de ce qui va bien et de ce qui laisse à désirer. On peut encore entendre le sens plus familier de la revue, l'occasion de se revoir, de se rencontrer, d'être de revue. La sociabilité conditionne la réussite de l'opération.

1 Chacune de ces rencontres a donné lieu à une publication. Saint-Maximin : Perrot 1984 ; Jussieu : Basch et al. 2001 ; Rouen : Sohn et Thélamon 1998.

CLIO, Histoire, Fermmes et Sociétés, 2002, 16, pp. 99-106. 
L'accueil chaleureux des Lyonnaises et l'éclat de leur ville illuminée (le 8 décembre est traditionnellement la fête des lumières à Lyon) venant agrémenter le sérieux des débats, ont donc fait de cette rencontre un événement, un jalon qui prend maintenant forme écrite, précisément dans la revue CLIO HSF.

L'exercice qui consiste à tirer les conclusions d'échanges polymorphes, à partir d'informations saisies au vol et non de textes lus, ne peut dégager que quelques points saillants qui ressortent de l'ensemble des interventions. Entre l'instantané de la rencontre et le moment de la rédaction, le recul invite aussi à réévaluer certaines impressions et à ouvrir d'autres pistes.

Une remarque préalable concerne la partition de la journée en deux tables rondes, distinguant les revues spécifiques et les revues généralistes. Cette organisation a donné l'impression, qui s'est confirmée au fil des débars, d'avoir affaire à deux univers autarciques, tendant à s'exclure l'un l'autre en dépit de la volonté de dialogue affichée par ailleurs. Ainsi la matinée consacrée aux revues d'histoire des femmes a dégagé une assurance à la fois tranquille et inquiète, confiante en sa légitimité, mais soucieuse de reconnaissance. L'après-midi réservé à la question de la place de I'histoire des femmes dans les revues généralistes est venu obscurcir l'horizon en pointant une sorte de no women's land dans l'espace scientifique. Cette tension peut se décliner en termes d'ignorance, de résistance ou de rattrapage. Ne pose-t-elle pas plus profondément la question du processus de légitimation ? Entre l'affrontement parfois peu amène et les forces d'attraction plus séduisantes, peuvent se jouer des relations complexes, instables, mobiles qui ménagent autant d'interstices pour des rencontres possibles. Prendre du recul n'invite-t-il pas aussi à décoder le jugement critique distillé par la communauté des pairs à travers la fabrique de leurs revues? Cette forme de production, qui n'est pas mineure pour la diffusion de la recherche $e^{2}$, s'avère être un révélateur précieux des interrogations

2 A titre indicatif, au Centre de Recherches Historiques par exemple, les articles de revues représentent $40 \%$ de la production bibliographique de ses chercheurs. Cette part relativement stable depuis trente ans est encore plus importante pour les femmes, surtout si elles appartiennent aux statuts " inférieurs " (comme les ingénieurs). 
qui traversent une profession, de ses pratiques et de la nécessaire clarification entre des activités de savoir, de mémoire et de pouvoir qui sont indissociables ${ }^{3}$.

\section{Conquête d'une autonomie professionnelle}

La table ronde de la matinée a réuni des revues de plusieurs pays européens, qui appartiennent à ce qu'on pourrait appeler la deuxième vague des revues d'histoire des femmes. Pénélope, portée par la première vague, fair figure d'ancêtre vénérable ; mais ses liens avec le mouvement féministe l'avaient enfermée dans une image militante et maintenue en marge du champ académique ${ }^{4}$. Le trait commun revendiqué par ses héritières, toutes nées dans les années 1990, est le professionnalisme, affirmé dans la forme et dans le fond, dans le fonctionnement et dans le souci de qualité scientifique. Sans doute l'ironie de certains titres (Sextant, L'Homme. Z.F.G.) évoque-t-elle le caractère souvent ludique des manifestes féministes, tandis que le terme de "femme " conserve sa valeur d'appel auprès d'un large public. Au-delà de l'image de marque affichée dans le titre, les revues présentent des traits communs : elles sont en majorité l'œuvre de femmes, bien que la non mixité ne soit pas érigée en principe ; arrimées aux structures universitaires et au monde de l'édition, elles mobilisent aussi des réseaux issus du féminisme ; les modes de production (comité de rédaction, comité de lecture, organisation des textes, comptes rendus, mise en page, tirage, diffusion...) s'apparentent à ceux des revues scientifiques en général.

Cette professionnalisation par rapport à la première époque de l'histoire des femmes s'accompagne d'un souci de pluralité, déjà revendiqué par les aînées : volonté d'associer toutes les disciplines des sciences humaines autour de l'histoire, ouverture internationale, proximité avec les témoignages et les questions du temps présent.

Du point de vue de l'extension chronologique couverte par le contenu des articles, on peut être frappé par la place faite au monde contem-

3 Noiriel 1996.

4 Dauphin 2001. 
porain au détriment des périodes anciennes. S'agit-il simplement de l'effet d'attraction opéré par la sociologie, l'anthropologie et les sciences politiques? La présence de ces disciplines pousse sans doute à réduire l'échelle du temps et à accorder plus d'attention à l'actualité. Des raisons documentaires liées au "silence " des femmes dans l'histoire peuvent aussi rendre moins accessibles des périodes éloignées et privilégier le témoignage direct. En outre, les approches interdisciplinaires mettent plus volontiers l'accent sur les phénomènes structurels que sur leur contextualisation.

Ne faudrait-il pas aussi s'interroger sur les relations entre le contenu des revues et le travail des historiennes ? Resterait à faire une véritable enquête sociologique sur les itinéraires et sur les contraintes qui les infléchissent : formation, carrière, choix des sujets de thèses et des thèmes de recherches, conditions pratiques du travail'5. Le découpage des objets est en partie tributaire des traits spécifiques du métier pour des chercheuses entrées récemment dans cette profession et surdéterminées par les modes de sélection et de reconnaissance ${ }^{6}$. À titre d'hypothèse, le fait que des femmes occupent majoritairement le champ de leur propre histoire ne renforce-t-il pas précisément la part de la période contemporaine dans les revues ? Par ailleurs, cette question sur la chronologie fait écho à celle, posée dès les débuts de l'histoire des femmes comme un défi, sur la recherche d'une nouvelle périodisation. Alors que les travaux de sociologie sur les temps sociaux mettent l'accent sur des différences de genre, l'historiographie des femmes continue de se couler dans le moule chronologique « classique " des siècles et des époques. Le thème de la temporalité, à savoir le vécu et les modes d'appropriation du temps en fonction des identités socioculturelles et sexuées, reste un domaine encore en friche que les revues pourraient inscrire dans leurs projets.

La professionnalisation se conquiert aussi par rapport à un environnement. Il ne suffit pas d'affirmer qu'un domaine de l'histoire appartient

5 "Profession : historienne ?", table ronde du 6 octobre 2001, organisée par Mnémosyne, association pour le développement de l'histoire des femmes et du genre (actes publiés dans le bulletin $n^{\circ} 1$, juin 2002).

6 Dumoulin in Sohn et Thélamon 1998 : 343-356. 
aux femmes, il faut aussi "forcer les portes de la reconnaissance institutionnelle " $"$. Du point de vue de la réception, les revues d'histoire des femmes s'adressent à la fois au milieu académique et au grand public. Elles s'inscrivent aux frontières de deux mondes qui se rencontrent souvent dans l'édition : à travers les femmes sont visés des problèmes de société (famille, mœurs, violence...). En fait, malgré cette position stratégique, les revues, encore jeunes, s'inquiètent de leur diffusion et de leur pérennité à la charnière de lectorats diversifiés. Les situations nationales different mais il est remarquable que chacune ait misé sur la production de numéros thématiques pour baliser le territoire de l'histoire des femmes. Cet habillage qui donne unité et cohérence à une collection d'articles permet en effet de renforcer la visibilité du champ. Au-delà des effets de mode, on voit poindre des thèmes novateurs : les pratiques sportives, les migrations, la violence, la sexualité, la paternité, les sciences et les techniques..., ou bien des thèmes qui demandent à être revisités : les religions, les classes sociales, la patrie, le corps... On note une certaine synchronie dans l'émergence des questions posées, du moins en Europe. Elles semblent s'imposer comme des évidences ou des urgences. Cependant le renouvellement nécessaire des objets ne peut faire l'économie d'une réflexion plus austère sur les méthodes et l'épistémologie. L'innovation en histoire réside sans doute plus dans la façon de questionner et de faire surgir du sens, que dans l'énoncé d'un thème.

\section{Cloisonnements}

Que proposent les revues généralistes sur l'histoire des femmes et du genre ? Qu'ont-elles proposé depuis la naissance de ce champ spécifique ? Il est apparu, au cours de cette table ronde, que les bilans quantitatifs oscillent dans une marge étroite allant de l'absence à la parcimonie (quelques articles par an). En fonction des thématiques proposées dans des numéros spéciaux (exemple : Le Mouvement Social en 1978 sur " Les travaux de femmes ") ou de la publication des actes d'un colloque (exemple : les Annales, E.S.C. en 1993 avec quatre " notes critiques"

7 Thébaud in Sohn et Thélamon $1998: 393$. 
issues du colloque Femmes et Histoire tenu à la Sorbonne en novembre 1992), les femmes ont pu faire une entrée remarquée, l'exception venant confirmer la règle de l'ignorance ou de la rareté.

Reste une autre entrée, la (petite) porte des recensions : elle ménage plus de flexibilité dans l'inscription de travaux sur les femmes et le genre. Ces fluctuations peuvent paraître aléatoires, elles ne répondent pas à des règles explicites, voire, elles n'ont guère, jusqu'à la journée de Lyon, questionné les rédactions qui se défendent de toute volonté d'exclusion, comme de tout postulat paritaire.

Le bilan ne peut se contenter d'une comptabilité froide, elle-même tributaire des index et des critères de classement. Les raisons invoquées pour justifier cet état de fait paraissent plus significatives. Il n'y a pas lieu ici de chercher à décrypter les lois silencieuses du milieu. Mais face à une situation de pénurie, on ne peut s'empêcher de suggérer quelques pistes.

Dans l'ordre pratique, les descriptifs du fonctionnement font apparaître une corrélation entre la présence de femmes au sein des comités et le contenu des revues. Le Mouvement Social illustre ce constat : elle est la seule pouvant se targuer d'avoir eu quatre femmes dans son équipe fondatrice (Annie Kriegel, Michelle Perrot, Madeleine Rébérioux et Rolande Trempé), elle fut et reste la plus ouverte aux recherches sur les femmes. Fruit d'un travail collectif, les revues mettent ce collectif en scène, dans l'assemblage de textes provenant d'horizons divers, dans le dispositif des recensions comme autant de chambres d'écho renvoyant à d'autres formes, d'autres lieux et d'autres points de vue. La fabrication d'une revue mobilise aussi, de façon moins visible, des réseaux directement issus du monde de la recherche : les choix et les découpages proposés dans chacun des numéros résultent d'un ensemble complexe de relations de pouvoir, souvent opaques pour les non initiés. Le déficit de femmes aux postes clés des décisions peut expliquer, de façon pragmatique, la fragile inscription de leur histoire dans le contenu.

Des raisons intellectuelles ont été invoquées : " résistance épistémologique " aux problématiques de genre ; réticences à repasser l'histoire au crible du genre, vu comme un principe explicatif unique ; critique du linguistic turn et du relativisme; assimilation de l'histoire des femmes à une 
histoire sociale qui aurait vocation de l'absorber ; discrédit du militantisme. Ces " raisons " n'ont guère été discutées au cours de la table ronde. On peut le regretter. Mais elles sont récurrentes et ne concernent pas seulement la fabrique des revues, elles interpellent toute la communauté historienne. L'histoire des femmes et du genre est-elle à ce point entachée de déficit explicatif, oublieuse de la complexité des sociétés et de la capacité des acteurs à subvertir le sens et les pratiques ? L'immense effort qui a permis d'extirper les femmes du continent noir de l'historiographie a sans doute, dans une première étape, édifié une cloison défensive autour d'un territoire conquis, parfois assimilé au ghetto. Mais depuis, que de chemin parcouru! On comprend mal que les systèmes interprétatifs ne puissent pas se croiser, là où il est précisément question de relations entre les hommes et les femmes, de masculinité autant que de féminité, de nation aussi bien que de famille, de compétences et de négociations autant que de codes et de représentations. Bref, on peut se demander en dernier ressort si les raisons pratiques ne sont pas plus résistantes à l'innovation que les « prétextes » épistémologiques.

De façon générale, cette journée confirme que la professionnalisation du métier d'hisrorien, comme l'a bien montré Gérard Noiriel${ }^{8}$, repose sur l'élaboration d'instruments de travail parmi lesquels la revue scientifique s'impose de façon décisive. Sorte d'outil polyvalent, flexible, elle permet de diffuser à la fois des travaux originaux et innovants, des références bibliographiques et des recensions de livres. Sa fonction ne se limite pas à la diffusion d'un savoir. Dans sa fabrique et dans sa réception, elle construit une communauté de lecteurs, elle tisse des liens, en fait prendre conscience, et rend sensible des solidarités. En ce sens, les années 1990 marquent une étape décisive dans le processus de professionnalisation $\mathrm{du}$ métier d'historienne, la naissance conjointe en Europe de différentes revues manifeste la grande vitalité de l'histoire des femmes. L'étape suivante - le besoin en devient pressant - consisterait à fonder les termes d'un échange intellectuel sur les problématiques de genre, autrement

8 Noiriel 1996. 
qu'en refoulant les dits articles vers les revues spécialisées. Faute d'accueil par les revues généralistes, l'histoire des femmes risque de s'installer et de s'isoler dans des espaces propres, dispensant ainsi les premières de s'impliquer dans le débat.

\section{Bibliographie}

BASCH Françoise, BRUIT Louise, DENTAL Monique, PICQ Françoise, SCHMITT PANTEL Pauline et ZAIDMAN Claude (sous la responsabilité de), 2001, Vingt-cinq ans d'études féministes : L'expérience Jussieu, (Journée du 14 novembre 1997), Cahiers du CEDREF, Université Paris 7 - Denis Diderot.

DAUPHIN Cécile, 1999, "Les publications, vitrine du Centre de Recherches Historiques ", communication au colloque Histoire de la recherche collective en sciences sociales au $X X^{2}$ siècle, 4-6 novembre 1999, à paraître.

__ 2001, "Pénélope : une expérience militante dans le monde académique ", in Vingt-cinq ans d'études féministes: l'expérience Jussieu, (Journée du 14 novembre 1997), Cahiers du CEDREF, Université Paris 7 - Denis Diderot, pp. 61-68.

DUMOULIN Olivier, 1998, "Archives au féminin, histoire au masculin. Les historiennes professionnelles en France, 1920-1965 ", in SOHN Anne-Marie et THELLAMON Françoise (dir.), L'Histoire sans les femmes estelle possible?, Paris, Plon.

NOIRIEL Gérard, 1996, Sur la "crise " de l'histoire, Paris, Belin.

PERROT Michelle (dir.), 1984, Une histoire des femmes est-elle possible? Marseille, Rivages.

SOHN Anne-Marie et THÉLAMON Françoise (dir.), 1998, L'Histoire sans les femmes est-elle possible ?, Paris, Plon.

THÉBAUD Françoise, 1998, "Conclusion ", in SOHN Anne-Marie et THÉLAMON Françoise (dir.), L'Histoire sans les femmes est-elle possible?, Paris, Plon, pp. 389-400. 